\title{
Positive cooperativity between acceptor and donor sites of the peptidoglycan glycosyltransferase
}

\author{
Daniel Bury ${ }^{\mathrm{a}, 1,2, *}$, Ismahene Dahmane ${ }^{\mathrm{b}, 2}$, Adeline Derouaux $^{\mathrm{b}}$, Shrinivas Dumbre ${ }^{\mathrm{c}}$, \\ Piet Herdewijn ${ }^{c}$, André Matagne ${ }^{\mathrm{b}}$, Eefjan Breukink ${ }^{\mathrm{d}}$, Erika Mueller-Seitz ${ }^{\mathrm{a}}$, Michael Petz ${ }^{\mathrm{a}}$, \\ Mohammed Terrak ${ }^{\mathrm{b}, *}$ \\ ${ }^{a}$ Department of Food Chemistry, Faculty of Mathematics and Natural Sciences, University of Wuppertal, Gaussstr. 20, 42119 Wuppertal, Germany \\ ${ }^{\mathrm{b}}$ Centre d'Ingénierie des Protéines, Université de Liège, Allée de la Chimie, B6a, B-4000, Sart Tilman, Liège, Belgium \\ ${ }^{\mathrm{c}}$ Laboratory of Medicinal Chemistry, Rega Institute for Medical Research, University of Leuven, Leuven, Belgium \\ ${ }^{\mathrm{d}}$ Membrane Biochemistry and Biophysics, Department of Chemistry, Faculty of Science, Utrecht University, Padualaan 8, 3584 CH Utrecht, The Netherlands
}

A R T I C L E I N F O

\section{Article history:}

Received 24 September 2014

Accepted 7 November 2014

Available online $\mathrm{xxx}$

\section{Keywords:}

Glycosyltransferase

Peptidoglycan

Moenomycin

SPR

Lipid II

\begin{abstract}
A B S T R A C T
The glycosyltransferases of family 51 (GT51) catalyze the polymerization of lipid II to form linear glycan chains, which, after cross linking by the transpeptidases, form the net-like peptidoglycan macromolecule. The essential function of the GT makes it an attractive antimicrobial target; therefore a better understanding of its function and its mechanism of interaction with substrates could help in the design and the development of new antibiotics.

In this work, we have used a surface plasmon resonance Biacore ${ }^{\mathbb{R}}$ biosensor, based on an amine derivative of moenomycin A immobilized on a sensor chip surface, to investigate the mechanism of binding of substrate analogous inhibitors to the GT. Addition of increasing concentrations of moenomycin A to the Staphylococcus aureus MtgA led to reduced binding of the protein to the sensor chip as expected. Remarkably, in the presence of low concentrations of the most active disaccharide inhibitors, binding of MtgA to immobilized moenomycin A was found to increase; in contrast competition with moenomycin A occurred only at high concentrations. This finding suggests that at low concentrations, the lipid II analogs bind to the acceptor site and induce a cooperative binding of moenomycin A to the donor site. Our results constitute the first indication of the existence of a positive cooperativity between the acceptor and the donor sites of peptidoglycan GTs.

In addition, our study indicates that a modification of two residues (L119N and F120S) within the hydrophobic region of MtgA can yield monodisperse forms of the protein with apparently no change in its secondary structure content, but this is at the expense of the enzyme function.
\end{abstract}

(c) 2014 Elsevier Inc. All rights reserved.

\section{Introduction}

The glycosyltransferases of family 51 (GT51) are essential enzymes found in bacteria with peptidoglycan cell wall [1]. They exist in two forms: as a monofunctional domain or linked to the N-terminal end of penicillin-binding (PB) domain in bifunctional PB proteins (PBPs) [1]. Both forms catalyze the polymerization of lipid II (undecaprenyl pyrophosphate-MurNAc(pentapeptide)-GlcNAc) precursor to form

\footnotetext{
* Corresponding authors.

E-mail addresses: bury@ipa-dguv.de (D. Bury), mterrak@ulg.ac.be (M. Terrak).

1 Present address: Institute for Prevention and Occupational Medicine of the German Social Accident Insurance, Institute of the Ruhr-Universität Bochum (IPA) Bürkle-de-la-Camp-Platz 1, 44789 Bochum, Germany. Tel.: +49 2343024796 , fax: +492343024605.

${ }^{2}$ D.B and I.D contributed equally to this work.
}

linear glycan chains which, after cross linking by the transpeptidases, form the net-like peptidoglycan macromolecule that encases bacteria and protects them from rupture under their high cytoplasmic pressure. Inhibition of the GT blocks peptidoglycan synthesis and leads to bacterial lysis and death, therefore the GT is a promising target in the search for new antibacterial agents to counter the arising antibiotic resistant strains.

Two main strategies are being followed to identify novel pharmacophore lead molecules for the design and synthesis of new GT inhibitors that could be developed as antibiotics: high throughput screening of chemical libraries [2-4] and rational synthesis of substrate and moenomycin (GT inhibitor) analogs [58]. The second strategy requires a deep understanding of the GT mechanism and structural data on protein-ligand complexes. Substantial progress has been made in the biochemistry of the GT [9-14] and several crystal structures have been determined, both 
in the apo form and in complex with moenomycin A and a lipid II analog [15-20]. These structures show that the GT is composed of a lysozyme-like globular domain and a small hydrophobic domain (called jaw domain) with a deep cleft between them containing the active site (Fig. 1). The extended active site is divided into two subsites, separated by a mobile region (composed of an alpha helix and a beta-hairpin), a donor site for the growing glycan chain binding and an acceptor site for lipid II binding. Elongation of the growing chain is achieved by the addition of disaccharide unit MurNAc-GlcNAc of lipid II in a processive way. Comparison of the apo and moenomycin A bound structures shows that the head domain remains unchanged after moenomycin A binding whereas a major conformational change occurs in the jaw subdomain with partial restructuration of the mobile region [15].

At the initiation phase of polymerization, the lipid II substrate molecules bind to the donor and acceptor sites which makes binding studies to determine the affinity of the substrate for each site particularly complex. Differences in affinity would determine the order of binding in the initiation stage and might consequently affect the affinity for the second site by an allosteric effect. We have used lipid II mimic inhibitors and moenomycin A, which is proposed to mimic the growing chain, to investigate these questions. An assay was developed by a modification of the SPR method described by Welzel and colleagues [21]. This technique is based on the interaction of an immobilized moenomycin A amino derivative with peptidoglycan glycosyltransferase and was originally used to test moenomycin derivatives for their inhibitory activity. We improved the sensitivity of this assay and used it to characterize several lipid II analog inhibitors as well as enzymatically inactive GT mutants as to their donor site functionality. Moenomycin A necessary for the synthesis of the amino derivative is not commercially available. Hence we also developed a method to isolate moenomycin A in good purity and yields from a Flavomycin ${ }^{\circledR}$ standard containing a mixture of moenomycin congeners.

We show that in the presence of low concentrations of lipid II analogs, supposedly bound to the acceptor site, moenomycin A binding to the donor site of the GT increased, indicating an allosteric activation of the donor site. At higher concentrations of these analogs, lower GT binding levels were observed, indicating competition with moenomycin A at the donor site.

All GTs contain a conserved hydrophobic surface (Fig. 1) that mediates their interaction with the cytoplasmic membrane and renders the purified proteins polydisperse [18,22]. This property makes a thorough quantitative binding study of the MtgA by SPR complex. The possibility to improve the solubility (monodispersity) of the protein by modification of residues in the hydrophobic surface was investigated. A monodisperse form of the protein was obtained without detergent and characterized.

\section{Materials and methods}

\subsection{Materials}

Surface plasmon resonance (SPR) biosensor analyses were performed on a Biacore $Q^{\circledR}$ (Biacore AB, Uppsala, Sweden). Semipreparative HPLC (high performance liquid chromatography) was performed using an HPLC consisting of a Merck-Hitachi LaChrom ${ }^{\circledR}$ Pump L7100 (Hitachi Ltd., Tokyo, Japan), a manual sample injector valve model 7125 (Rheodyne Inc., Cotati, USA), a NUCLEODUR ${ }^{\circledR}$ C18 HTec column (dimensions: $250 \times 10 \mathrm{~mm}$, particle size: $5 \mu \mathrm{m}$ ) (Macherey-Nagel GmbH \& Co. KG, Düren, Germany) in a thermostated column compartment model ERC 125 (ERC GmbH, Riemerling, Germany), a Merck-Hitachi LaChrom ${ }^{\circledR}$ UV-vis Detector L-7420 with semi-micro flow cell (Hitachi Ltd., Tokyo, Japan), and a 162 chromatography signal interface (Autochrom Inc., Milford, USA). Analytical HPLC was performed using an HPLC consisting of a Merck-Hitachi LaChrom ${ }^{\circledR}$ Pump L7100 (Hitachi Ltd., Tokyo, Japan), a Merck-Hitachi LaChrom ${ }^{\mathbb{R}}$ Programmable Autosampler L-7250 (Hitachi Ltd., Tokyo, Japan), a NUCLEODUR ${ }^{\circledR}$ C18 HTec column (dimensions: $250 \times 4 \mathrm{~mm}$, particle size: $5 \mu \mathrm{m}$ ) (Macherey-Nagel GmbH \& Co. KG, Düren, Germany) in a Merck LaChrom $^{\circledR}$ Column Oven L-7350 (Merck KGaA, Darmstadt, Germany), a Merck-Hitachi LaChrom ${ }^{\circledR}$ Diode Array Detector L7450 (Hitachi Ltd. Tokyo, Japan), and a Merck-Hitachi Interface D7000 (Hitachi Ltd., Tokyo, Japan). HPLC eluents and SPR running buffers were filtered through a Whatman ${ }^{\circledR} \mathrm{OE} 67$ cellulose acetate membrane filter (pore size $0.45 \mu \mathrm{m}$; Whatman $\mathrm{GmbH}$, Dassel, Germany) and degassed prior to use.

High-purity water was produced from deionized water using a Milli- $Q^{\circledR}$ Gradient A10 (Millipore, Molsheim, France). Acetonitrile (Fisher Scientific UK Limited, Loughborough, UK), methanol (VWR International, Fontenais-Sous-Bois, France), acetone (VWR International, Fontenais-Sous-Bois, France), and chloroform (Fisher Scientific UK Limited, Loughborough, UK) were HPLC grade. Acetonitrile (puriss.) for semi-preparative HPLC, dimethyl sulfoxide (p.a.), 25\% ammonia, sodium hydroxide ( $\geq 99 \%$ ), sodium chloride (p.a.), HEPES (p.a.), TRIS ( $\geq 99.9 \%$ ), and EDTA (disodium
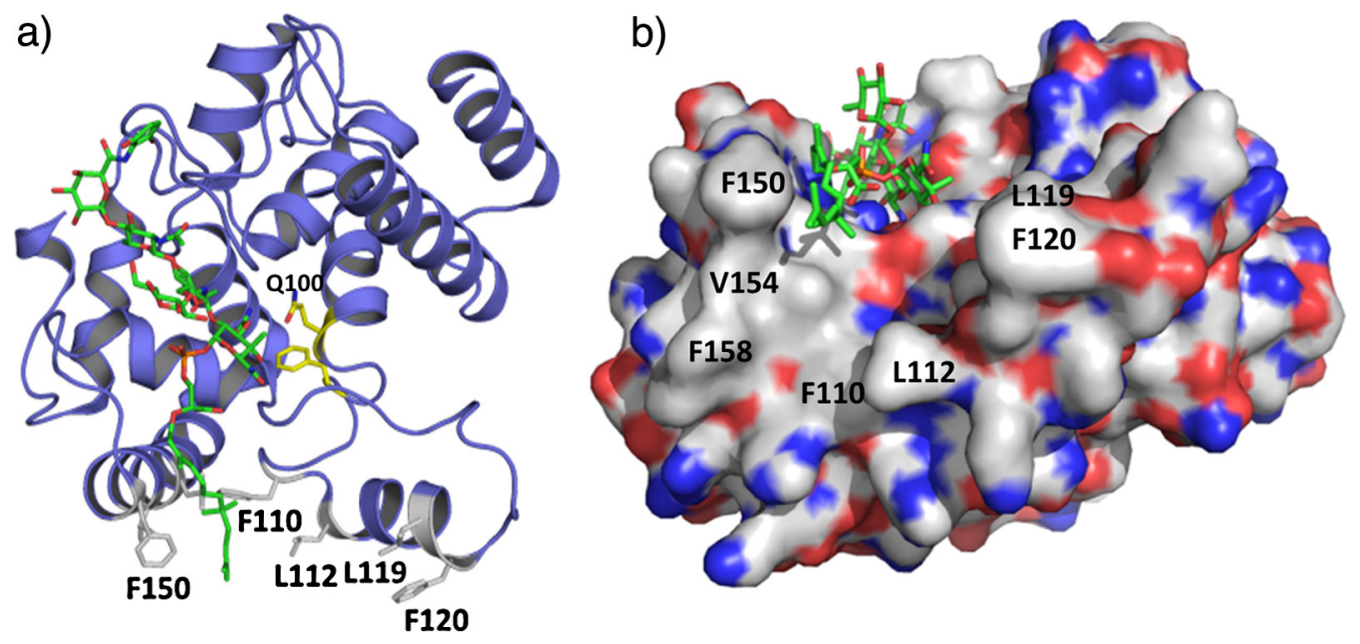



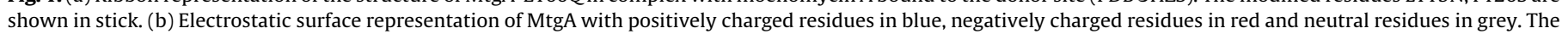
residues of the hydrophobic surface are indicated. Figures were generated by The PyMOL Molecular Graphics System, Version 1.5.0.4 Schrödinger, LLC). 
salt, dihydrate; p.a.) were purchased from Carl Roth $\mathrm{GmbH}+\mathrm{Co}$ (Karlsruhe, Germany). Ethanolamine ( $\geq 98 \%$ ), potassium dihydrogen phosphate (puriss, p.a.), anhydrous dipotassium hydrogen phosphate (purum, p.a.), TWEEN ${ }^{\circledR} 20$, SDS ( $\geq 99.0 \%$ ), 5-amino-2nitrobenzoic acid (97\%), 1,1'-carbonyldiimidazole (>90\%), $\mathrm{N}-(3-$ dimethylaminopropyl)- $N^{\prime}$-ethylcarbodiimide hydrochloride (purum) and $\mathrm{N}$-hydroxysuccinimide (98\%) were purchased from Sigma-Aldrich Chemie GmbH (Steinheim, Germany). 85\% orthophosphoric acid (p.a.), ammonium hydrogen carbonate (puriss.), sodium acetate trihydrate (p.a.) and sodium nitrite (p.a.) were purchased from Merck KGaA (Darmstadt, Germany). Anhydrous pyridine (p.a.) was obtained from Fluka (Seelze, Germany), hydrochloric acid ( 36\%) from Fisher Scientific UK Limited (Loughborough, UK), and CHAPS $(\geq 98.0 \%)$ from A.G. Scientific, Inc. (San Diego, USA). Flavomycin ${ }^{\circledR}$ reference standard was purchased from biovet ${ }^{\circledR}$ (Peshtera, Bulgaria).

Unlabled lipid II was prepared as described [23]. The syntheses of lipid II analogs 21, 43, 44, 56, 57 61, 62 have been described in [5].

\subsection{Preparation of moenomycin $A$}

Moenomycin A was isolated from the Flavomycin ${ }^{\circledR}$ reference standard as previously reported [24] by semi-preparative HPLC followed by desalting via SPE. The HPLC separation was performed with $6 \mathrm{~mL} / \mathrm{min} 50 \mathrm{mM} \mathrm{KH} \mathrm{PO}_{4} \mathrm{pH}$ 2.3/acetonitrile 58:42 (v/v) at $45^{\circ} \mathrm{C}$ and a detection wavelength of $280 \mathrm{~nm}$. With each chromatographic run approximately $35 \mathrm{mg}$ Flavomycin ${ }^{\mathbb{R}}$ in $100 \mu \mathrm{L}$ water/acetonitrile $8: 2(\mathrm{v} / \mathrm{v})$ were injected. Fractions containing moenomycin A were analyzed via analytical HPLC $\left(1 \mathrm{~mL} / \mathrm{min} 100 \mathrm{mM} \mathrm{NH}_{4} \mathrm{HCO}_{3} \mathrm{pH} 8.0 /\right.$ acetonitrile 55:45 (v/v) at $45^{\circ} \mathrm{C}$ ) and chromatographically pure moenomycin A fractions were combined, diluted $1: 2$ with $50 \mathrm{mM}$ phosphate buffer $\mathrm{pH} 7.0$ (to improve retention on the SPE phase) and desalted via SPE on CHROMABOND $^{\circledR} \quad$ HR-X SPE cartridges (1000 mg sorbent) (Macherey-Nagel GmbH \& Co. KG, Düren, Germany). After conditioning with methanol, followed by water and $50 \mathrm{mM}$ phosphate buffer pH 7.0 (15 mL each), on each SPE cartridge the combined moenomycin A fractions from three chromatographic separations (corresponding to approximately $100 \mathrm{mg}$ Flavomy$\operatorname{cin}^{\mathbb{R}}$ ) were loaded with $10 \mathrm{~mL} / \mathrm{min}$. After washing with water (3 times $5 \mathrm{~mL}$ ), moenomycin A was eluted with acetone/water 6:4 $(\mathrm{v} / \mathrm{v}$ ) with $1 \mathrm{~mL} / \mathrm{min}$ at maximum (2 times $10 \mathrm{~mL}$ have proven to be sufficient). Acetone was removed via rotary evaporator and the remaining solutions were lyophilized.

\subsection{Synthesis of moenomycin A amino derivative}

5-Amino- $\mathrm{N}$-(2-amino-ethyl)-2-nitrobenzamide was synthesized according to Stembera et al. [25] except for the activated 5-amino-2-nitrobenzoic acid being slowly added to the cooled solution of ethylene diamine and the product being purified by column chromatography on silica gel (washed with $\mathrm{HCl}$ and the water content set to $1.5 \%(\mathrm{w} / \mathrm{w})$ according to [26]) with $25 \%$ ammonia/methanol 1:25 (v/v).

Compound $3 \mathrm{~b}$ in [25] was synthesized from $100 \mathrm{mg}$ moenomycin A according to Stembera et al. [25] except that no shield gas was used and except for cleanup, which was performed by SPE on CHROMABOND ${ }^{\circledR}$ HR-X SPE cartridges (3 cartridges with $1000 \mathrm{mg}$ sorbent each) (Macherey-Nagel GmbH \& Co. KG, Düren, Germany) after adjusting the $\mathrm{pH}$ to 7.5 (the amino derivative is poorly soluble in acidic solution). After washing with $50 \mathrm{mM} \mathrm{K}_{2} \mathrm{HPO}_{4} \mathrm{pH} \mathrm{7.5/}$ acetonitrile $8: 2(\mathrm{v} / \mathrm{v})$ (2 times $5 \mathrm{~mL}$ per cartridge) and subsequent washing with water ( $5 \mathrm{~mL}$ per cartridge), the product was eluted with acetone/water $6: 4(\mathrm{v} / \mathrm{v})$ ( 4 times $10 \mathrm{~mL}$ per cartridge). Acetone was removed via rotary evaporator and the remaining solution was lyophilized to yield $87 \mathrm{mg}$ of the desired product. Identity was confirmed by its measured accurate mass (negative mode ESI-ToF-MS). The residual moenomycin A content was determined via HPLC with $1 \mathrm{~mL} / \mathrm{min} 50 \mathrm{mM} \mathrm{KH}_{2} \mathrm{PO}_{4} \mathrm{pH} 2.3 /$ acetonitrile 47:53 (v/v) after dissolving an aliquot of the product in the eluent mixture and membrane filtration (CHROMAFIL ${ }^{\circledR}$ Xtra PET-45/25 (Macherey-Nagel GmbH \& Co. KG, Düren, Germany) to remove undissolved product. Residual content of 5-amino- $\mathrm{N}-(2-$ amino-ethyl)-2-nitrobenzamide was determined by HPLC with $1 \mathrm{~mL} / \mathrm{min} 100 \mathrm{mM} \mathrm{NH} \mathrm{HCO}_{3} /$ acetonitrile 95:5 (v/v) (detection wavelength: $380 \mathrm{~nm})$. The product contained $4 \%(\mathrm{w} / \mathrm{w})$ moenomycin $\mathrm{A}$ and $<0.1 \%$ (w/w) 5-amino- $\mathrm{N}$-(2-amino-ethyl)-2-nitrobenzamide (corresponding to $<1 \%$ mole fraction).

\subsection{Preparation of SPR biosensor chip surface}

The moenomycin A amino derivative was tethered to the surface of a Sensor Chip CM5 (GE Healthcare Bio-Sciences AB, Uppsala, Sweden) as described by Stembera et al. [25].

\subsection{Direct SPR biosensor assay for determining glycosyltransferase binding activity}

The glycosyltransferases (GT) were diluted in SPR running buffer ( $25 \mathrm{mM}$ Tris buffer $\mathrm{pH} 7.5$ containing $300 \mathrm{mM} \mathrm{NaCl}$ and $0.3 \%$ CHAPS) and injected over the sensor chip surface for $120 \mathrm{~s}$. Regeneration was performed by injection of 1\% SDS in running buffer for $30 \mathrm{~s}$ followed by a $60 \mathrm{~s}$ injection of pure running buffer. All steps were performed at a flow rate of $40 \mu \mathrm{L} / \mathrm{min}$. For each GT a dilution series was analyzed.

Since a CHAPS molecule was found in the crystal structure of PBP1a [20], we verified its effect on the GT activity and found no effect for up to 2 times the concentration used in the assay (data not shown), showing that CHAPS does not interfere with our assay.

The SPR biosensor used for our experiments cannot simultaneously inject a solution over different chip surfaces, thus subtraction of data from a reference cell could not be performed in real-time. To compensate possible baseline shift during analysis, sensorgrams are expressed as relative response values (actual response minus response $10 \mathrm{~s}$ before main injection of the respective cycle) versus time instead of absolute response values versus time (the same applies to Section 2.6).

\subsection{Competitive SPR biosensor assay on inhibitor activity}

For each inhibitor a dilution series was prepared in running buffer ((25 mM Tris buffer pH 7.5; $300 \mathrm{mM} \mathrm{NaCl}) /$ DMSO 9:1 (v/v) containing $0.3 \%$ CHAPS). The solutions were mixed with a stock solution of Staphylococcus aureus MtgA wild type (in running buffer) resulting in a final MtgA concentration of $400 \mathrm{nM}$. Each of these mixtures was injected over the sensor chip surface for $150 \mathrm{~s}$ with a flow rate of $10 \mu \mathrm{L} / \mathrm{min}$. GT binding levels were calculated from the response $30 \mathrm{~s}$ after the end of the injection (to eliminate the bulk effect of the solution) minus the response $10 \mathrm{~s}$ before start of the injection (to account for baseline shift in the course of the analysis). Regeneration was performed by injection of $1 \%$ SDS in running buffer for $30 \mathrm{~s}$ followed by a $60 \mathrm{~s}$ injection of pure running buffer (both steps at $40 \mu \mathrm{L} / \mathrm{min}$ ).

\subsection{Site-directed mutagenesis and purification of proteins}

Single and double mutations were generated using QuickChange site directed mutagenesis method (Agilent Technologies) using the oligonucleotides in Table 1 and the plasmid pDML2004 as template [27]. All constructs were checked by sequencing. For the exchange of 6 amino acids (MtgA-6M: L112N, L119N, F120S, 
Table 1

Sequences of oligonucleotides used for mutagenesis.

\begin{tabular}{ll}
\hline$m t g A$ mutants & Oligonucleotides \\
\hline L119N-F120S & 5'-GGTACAACTAGAGTCAACTCTTCAACGATTAGCGAC-3' \\
& 5'-GTCGCTAATCGTTGAAGAGTTGACTCTAGTTGTACC-3' \\
F120S & 5'-CTGTCGCTAATCGTTGAAGATAAAGCTCTAGTTGTAC-3' \\
& 5'-GTACAACTAGAGCTTTATCTTCAACGATTAGCGACAG-3' \\
F150S & 5'-GATAATGATCGCAGCTTCACCCGCAAAAGCAAAG-3' \\
& 5'-CTTTGCTTTTGCGGGTGAAGCTGCGATCATTATC-3' \\
F104A & 5'-CAATGGAAGATGAACGAGCCTACAATCATCATGGAT-3' \\
& 5'-GTTACCTTCTACTTGCTCGGATGTTAGTAGTACCTA-3' \\
\hline
\end{tabular}

F150S, V154S, F158S), a modified $m \operatorname{tg} A$ gene was ordered from GeneArt (Life technologies).

Escherichia coli PBP1b was expressed and purified using the pDML924 plasmid as previously described [11].

$S$. aureus MtgA and its mutants were prepared, purified and their activities tested as previously described [27]. MtgA mutants were purified under the same conditions as the wild type unless indicated. All the MtgA variants used are without transmembrane (TM) segment, this deletion does not affect the structure of the enzyme [18]. Deletion of the TM region was found to reduce the activity of the enzyme, most probably because TM contributes to the hydrophobic interaction with the substrate [19]. This construct is more suited for our studies because it will better highlight the specificities of the enzyme for the polar regions (sugars, peptide and phosphates) of substrate analogs.

\subsection{Analysis of MtgA and mutants by gel filtration}

Gel filtration of MtgA and mutants MtgA-NS and MtgA-F120S ( $2 \mathrm{mg} / \mathrm{mL}$ ) was performed on a $24 \mathrm{~mL}$ Superdex-75 column (GE healthcare) in buffer $25 \mathrm{mM}$ Tris- $\mathrm{HCl} \mathrm{pH} 8,500 \mathrm{mM} \mathrm{NaCl}, 10 \%$ glycerol using Akta Explorer (GE healthcare). Protein fractions were collected and analyzed by SDS-PAGE.

\subsection{Circular dichroism (CD)}

Far-UV CD spectra (200-260 nm) were recorded with a Jasco J810 spectropolarimeter at $20^{\circ} \mathrm{C}$ in $25 \mathrm{mM}$ Tris- $\mathrm{HCl} \mathrm{pH} 8,500 \mathrm{mM}$ $\mathrm{NaCl}$, using a $1 \mathrm{~mm}$ pathlength quartz Suprasil cell (Hellma), with protein concentrations of $c a .0 .1 \mathrm{mg} / \mathrm{mL}$ (i.e. $4 \mu \mathrm{M}$ ). Four scans $(10 \mathrm{~nm} / \mathrm{min}, 1 \mathrm{~nm}$ bandwidth, $0.1 \mathrm{~nm}$ data pitch and $1 \mathrm{~s}$ DIT) were averaged, base lines were substracted and no smoothing was applied. Data are presented as the residue ellipticity $\left([\theta]_{\mathrm{MRW}}\right)$, calculated using the molar concentration of protein and number of residues.

\subsection{Large unilamellar vesicle (LUV) preparation and interaction with MtgA-WT and variants}

E. coli lipids extract $(20 \mathrm{mg})$ solutions in chloroform (Avanti Polar lipids Inc., Alabama, USA) were mixed and evaporated under a gentle stream of nitrogen. The lipid film was subsequently dried for 20 min under vacuum. The film was hydrated by the addition of the buffer ( $25 \mathrm{mM}$ Hepes pH 7.5, $150 \mathrm{mM} \mathrm{NaCl}, 10 \mathrm{mM} \mathrm{MgCl}$ ) under mechanical agitation and submitted to 10 freeze-thaw cycles using liquid nitrogen and a water bath. The lipid suspension was then extruded 10 times through a polycarbonate membrane filter with a pore size of $200 \mathrm{~nm}$ (Whatman International).

MtgA and variants at $80 \mu \mathrm{M}$ or lysozyme used as control were preincubated with or without LUV for $30 \mathrm{~min}$ at $4{ }^{\circ} \mathrm{C}$ under gentle mixing. The mixture was ultracentrifuged at $122,000 \times g, 14{ }^{\circ} \mathrm{C}$ for $30 \mathrm{~min}$. The supernatant and pellet fractions were separated and the pellet was washed 3 times in $25 \mathrm{mM}$ Tris buffer $\mathrm{pH} 8$, containing $300 \mathrm{mM} \mathrm{NaCl}$ following the same procedure. Fractions recovered after each centrifugation step were analyzed by SDSPAGE and the amount of bound (pellet) and unbound (supernatant) proteins were estimated.

\section{Results}

\subsection{Isolation of moenomycin A from the mixture of congeners}

Moenomycin A was obtained as a colorless powder with a purity >99\% (HPLC-UV, $258 \mathrm{~nm}$, Fig. 2). Due to the use of basic buffer as eluent for the analytical HPLC instead of acidic buffer (as used for semi-preparative HPLC), the elution order is changed making it easy to identify any remaining moenomycin $A_{12}$ in the isolated moenomycin A. Overall $225 \mathrm{mg}$ moenomycin A were isolated from $1050 \mathrm{mg}$ Flavomycin ${ }^{\circledR}$. By HPLC-UV we determined a moenomycin A content of $30 \%$ in the starting material, accordingly the yield was $>70 \%$. Identity of the isolated moenomycin A was confirmed by ${ }^{1} \mathrm{H}$ NMR (conditions as described by Hennig et al. [28]) and ESI-MS in negative mode (data not shown).

\subsection{Optimization of the SPR assay and analysis of GT binding to moenomycin A}

Moenomycin A inhibits the transglycosylation step by binding to the donor site of the glycosyltransferase. Thus, the ability of a GT to bind moenomycin A corresponds to its ability to bind its natural donor substrate (i.e. the growing glycan chain) and indicates that the donor site is correctly folded and functional.

Stembera et al. [21,25] described an SPR biosensor assay utilizing a moenomycin $\mathrm{A}$ amino derivative tethered to the chip surface and successfully applied it to test different moenomycin A analogs for their relative affinity towards E. coli PBP1b [21] (the analogs in solution compete with immobilized moenomycin A for the GT donor sites). We have optimized this assay by comparing PBP1b and S. aureus MtgA and found conditions, where MtgA was more sensitive and data more reproducible than with PBP1b (probably due to the presence of the transmembrane segment in PBP1b and requirement of higher detergent concentrations for solubility). Under these conditions moenomycin A at concentrations as low as $0.5 \mu \mathrm{M}$ caused a clear inhibition of MtgA binding (Fig. 3a). In Stembera et al. [21] $100 \mu \mathrm{M}$ moenomycin A clearly inhibited PBP1b binding, whereas $10 \mu \mathrm{M}$ did not cause any inhibition. We reproduced their experiments and confirmed this lower sensitivity for PBP1b. Therefore MtgA was chosen to analyze its interaction with substrate analogs.

First, 2-fold dilution series of MtgA from two different batches ranging from $125 \mathrm{nM}$ to $2 \mu \mathrm{M}$ were used to analyze its binding to immobilized moenomycin A. Clear concentration dependent binding responses were observed (Fig. 3b). However, the sensorgrams obtained cannot be described by a single exponential function (yet this should be possible for a simple 1:1 interaction without mass transport limitation [29]). They do not reach a steady state for any concentration (thus equilibrium analysis cannot be performed to calculate dissociation constants). Instead, after a first exponential phase, the binding curves reach a phase with practically constant (yet in comparison flat) slope, which cannot be explained by a $1: 1$ interaction. This complexity in the binding responses was also observed on a flow cell with low ligand density (310 RU moenomycin A amino derivative compared to $720 \mathrm{RU}$ for the other experiments) and even at low MtgA concentrations (Fig. 3c). Even here and despite the prolonged injection (300 s instead of $120 \mathrm{~s}$ ) a steady state was not reached. Since non-specific binding of GTs was not observed at concentrations below $5 \mu \mathrm{M}$, we presume this complexity of binding responses is caused by aggregation of GTs to one another after reaching a critical concentration on the chip surface most likely via the hydrophobic 

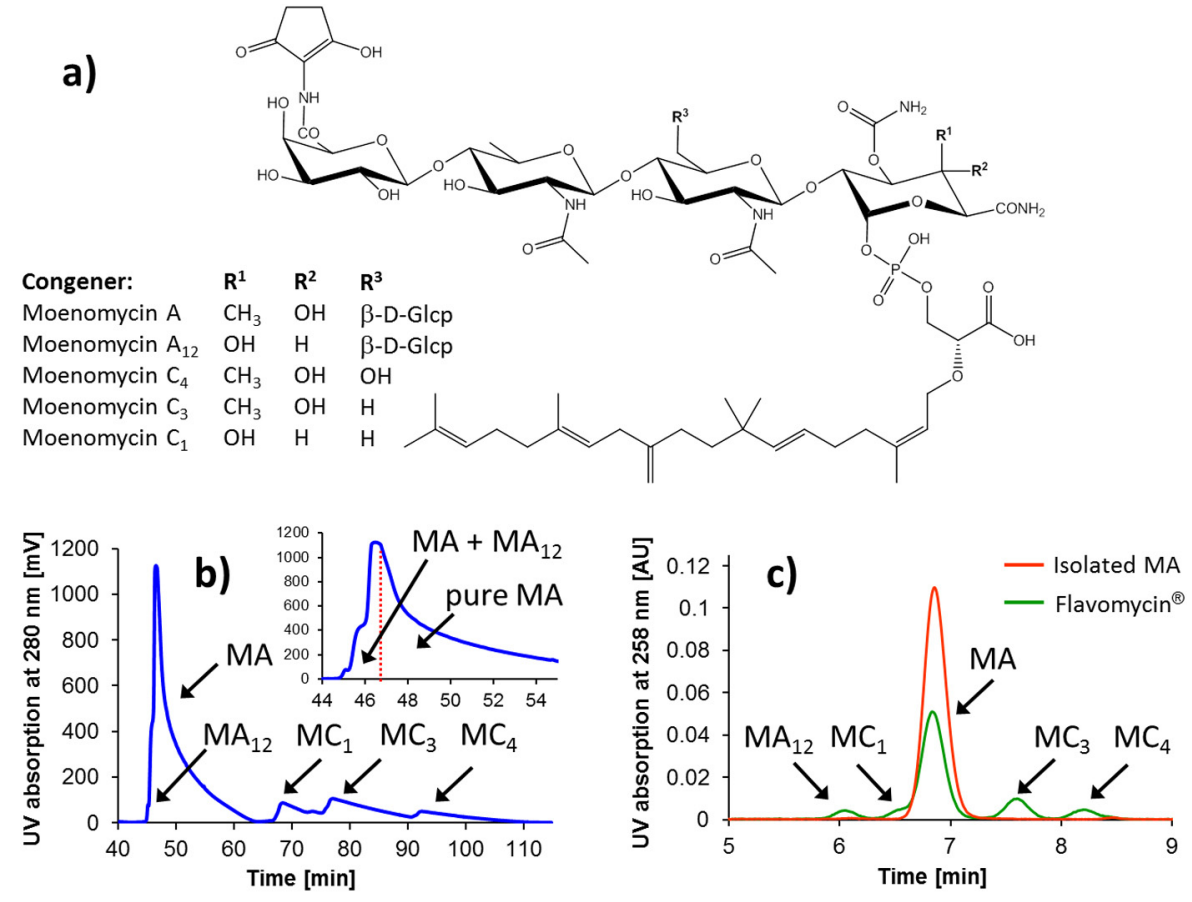

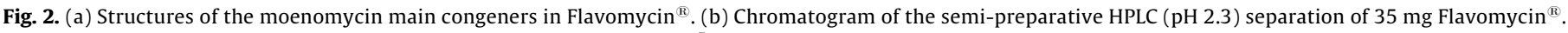

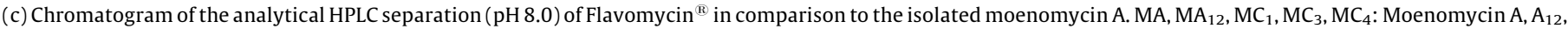
$\mathrm{C}_{1}, \mathrm{C}_{3}, \mathrm{C}_{4}$.

jaw region (attempts to improve solubility of MtgA have been investigated; see below). Since rate constants values derived from such complex binding data are known to provide ambiguous results [30], we do not present any value for association and dissociation rate constants characteristic of the interaction between the GTs and moenomycin A, but instead limit ourselves to qualitative or at most semi-quantitative conclusions.

\subsection{Modification of the hydrophobic region in MtgA and characterization of the mutants}

The GTs are characterized by a hydrophobic surface that mediates the interaction of the proteins with the cytoplasmic membrane (Fig. 1b). MtgA devoid of its transmembrane (TM) segment has tendency to aggregate due to its hydrophobic region. This behavior and the need of high concentrations of detergent prevent from detailed kinetic and binding characterization in vitro. We have tried to reduce the polydispersity of the protein through mutagenesis of the residues in the hydrophobic surface to facilitate in vitro study by SPR. In MtgA, this surface includes F110, L112, L119, F120, F150, V154, L157, F158. The following single mutations
F143T, V154T, L157T or F158T have no effect on the activity of MtgA but did not improve the solubility of the protein significantly [18]. F110 may be essential for binding of lipid II and its modification would affect activity [18]. We have prepared the following single mutations F120S and F150S, the double mutation L119N-F120S (MtgA-NS) and the multiple mutations simultaneously MtgA-6M (L112N, L119N, F120S, F150S, V154S, F158S). The mutant F150S and MtgA-6M could not be expressed in soluble form (e.g. presence in the supernatant fraction without detergent after cell lysis and centrifugation) using different expression conditions $\left(18-37^{\circ} \mathrm{C}\right)$. Since a Phe at position 150 seems to be important for proper folding of the protein, another mutant of all 6 residues, but F150 was prepared and also found insoluble.

The single mutant F120S and the double mutant L119N-F120S could be expressed in soluble form. Analysis of the double mutant $(2 \mathrm{mg} / \mathrm{mL}$ ) by gel filtration without detergent showed the protein eluted as a monomer in a single peak without any aggregate (Fig. 4a). Under these conditions the wild type MtgA was entirely found in the void volume with high molecular mass (Fig. 4a). Without detergent, monodispersity of the single mutant F120S was in between that of wild type MtgA and MtgA-NS. In the





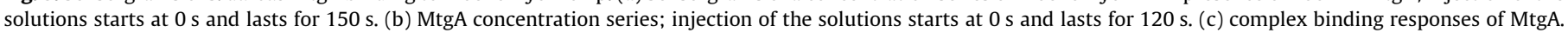


a)


Fig. 4. Comparative analysis of MtgA and the mutants MtgA-NS and F120S by gel filtration and circular dichroism. (a) Size exclusion profile of MtgA, mutants MtgANS and F120S on Superdex 75 column. (b) Far UV CD spectra of MtgA, mutants MtgA-NS and F120S.

presence of CHAPS about $80 \%$ of the wild type protein was monodisperse and 20\% aggregate (data not shown). Despite its monodispersity MtgA-NS was still able to bind lipid vesicles (LUV) (data not shown). Far-UV circular dichroism (CD) comparison of MtgA-NS and MtgA-F120S with the wild type MtgA showed similar spectra (Fig. 4b), characteristic of $\alpha$-helices profile with two characteristic minima at $208 \mathrm{~nm}$ and $222 \mathrm{~nm}$, in agreement with the protein structure. This result suggests that the mutation did not modify the secondary structures of the protein significantly. The mutants MtgA-NS and MtgA-F120S were unable to convert radioactive lipid II substrate to polymeric peptidoglycan even at high protein concentration indicating that they were inactive. Previous results showed that the MtgA mutant E100Q which retains $0.2 \%$ of the wild type activity binds to moenomycin $A$ $[18,27]$. Comparison of E100Q with another MtgA mutant F104A ( $1 \%$ of wild type activity) by SPR (at $400 \mathrm{nM}$ ), showed that E100Q binds moenomycin $A$ in the same order of magnitude as the wild type while the binding response for F104A was drastically decreased (Fig. 5a). Binding responses of both mutants were specific via their donor site as was tested by analyzing GT binding to the chip surface in presence of moenomycin A (data not shown). This data shows that inactive or barely active MtgA mutants could still be able to bind moenomycin A indicating that at least the donor site is functional. To test this possibility in the case of the most soluble mutant L119N-F120S, we studied its interaction with moenomycin A using SPR.

When tested at $125 \mathrm{nM}$ to $2 \mu \mathrm{M}$, MtgA-NS did not show any binding responses, it was then analyzed at higher concentrations
(Fig. 5b and c; additional experiment with double referencing: Fig. $5 \mathrm{~d}-\mathrm{f}$ ). The binding responses were rather random than concentration dependent, strongly suggesting non-specific binding of MtgA-NS to moenomycin A (e.g. binding of hydrophobic regions of the GT to the moenocinol moiety) (Fig. 5). Hence the amino acid exchanges L119N and F120S in the membrane binding surface of MtgA resulted in monodisperse protein and a complete loss of specific moenomycin A binding capability. These results suggest that the hydrophobicity of this region is important to maintain the correct structure of the jaw subdomain and/or for the interaction with the substrate and moenomycin. Another possibility could be that the two mutations (L119N, F120S) may affect the outer helix transition/conformational change during catalysis.

\subsection{Positive cooperativity between the acceptor and donor sites of the GT}

Several analogs of GT's lipid II substrate $(21,43,44,56,57,61$, and 62) (Fig. 6) have been synthesized previously and found to inhibit the GT activity in vitro and cause bacterial growth defect [5]. These compounds are valuable tools for understanding the complex catalytic mechanism of the GT. However, their precise mode of action remains elusive, such as their specificity for the donor and/or acceptor sites. In order to answer these questions we have analyzed their effect on moenomycin A binding of MtgA using SPR. In this assay, inhibitors capable of binding to the donor site of GTs should compete for this binding site with the moenomycin A amino derivative tethered to the sensor chip surface, resulting in a decreased GT binding response (Fig. 3a).

Mixtures of MtgA with varying concentrations of the compounds were tested for their effect on MtgA binding to a sensor chip surface modified with the moenomycin A amino derivative. In addition, the natural substrate lipid II (Fig. 6) was analyzed in the presence of EDTA in the running buffer to avoid polymerization. All substances were analyzed in 3-fold dilution series and measured in duplicate. For inhibitors 21, 43, 44, and 62 the concentration range was $7 \mathrm{nM}$ to $400 \mu \mathrm{M}$, for inhibitors 57 and 61 it was $3 \mathrm{nM}$ to $200 \mu \mathrm{M}$, for inhibitor 56 it was $3 \mathrm{nM}$ to $148 \mu \mathrm{M}$, and for lipid II $2 \mathrm{nM}$ to $120 \mu \mathrm{M}$. All substances tested showed inhibition of MtgA binding at high concentrations (Fig. 7a-d). Interestingly, at concentrations below approximately $50 \mu \mathrm{M}$, the disaccharide analogs 21, 44, and 62 clearly showed an increased binding response in comparison to MtgA in absence of inhibitor (Fig. 7a). Inhibitor 57 (below approximately $10 \mu \mathrm{M}$ ) and lipid II might have the same enhancement effect, but to a lesser extent (Fig. 7c and d). In case of lipid II, the binding responses had a high spread, preventing a thorough interpretation, most likely due to poor solubility. Enhancing solubility of lipid II by increasing the DMSO content in the running buffer did result in a drastic change in refractive index leading to SPR angles outside the detector range. Also, EDTA is known to form complexes with moenomycins [31] which might add to the poor quality of the data. The monosaccharide analogs 43, 56, and 61 clearly showed a concentration dependent inhibition but increased binding was not observed with these compounds (Fig. $7 \mathrm{~b}$ ), thus indicating a simple competition with moenomycin A for the donor site of the GT. Non-specific binding of the inhibitors to the sensor chip surface can be ruled out as a cause for the increased binding behavior, because it would not explain the decrease of binding responses at higher inhibitor concentrations. From the structural similarity of the disaccharide analogs with lipid II substrate they are expected to bind to the acceptor site of MtgA. Therefore, we suggest that at low concentrations the disaccharide compounds bind selectively to the acceptor site and increase the affinity of the donor site to moenomycin A by heteroallosteric activation leading to an increased MtgA binding response. This effect is rivaled by the 



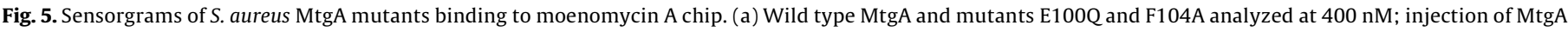

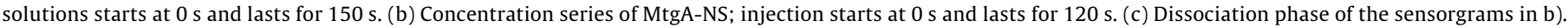

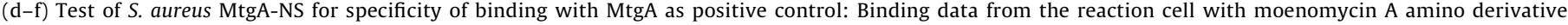

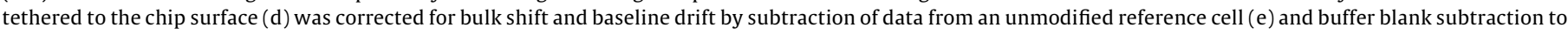

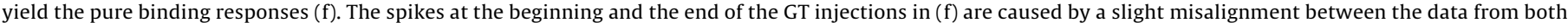
chip surfaces.

ability of the inhibitors to bind to the GT's donor site at higher concentration, which explains the decrease in the binding responses (competition for the donor site outweighs the enhanced binding). Such an allosteric activation would affect the association and/or dissociation rates for interactions with the donor site. Fig. 8 shows an overlay of sensorgrams for inhibitor 62 in the concentration range around the maximum of MtgA binding. The highest binding response was obtained at an inhibitor concentration of $4.9 \mu \mathrm{M}$. From there on the binding responses decreased both with increasing and decreasing inhibitor concentration. Since dissociation of the GT-inhibitor complexes follows a first order kinetic (i.e. dependent on the complex' concentration, $\mathrm{d}[L A] /$ $\left.\mathrm{d} t=-k_{d} \times[L A]\right)$, the sensorgram for the highest GT binding response $(4.9 \mu \mathrm{M})$ should have the steepest slope in dissociation phase, unless $k_{d}$ had changed. The sensorgrams for the two highest inhibitor concentrations ( 44 and $133 \mu \mathrm{M}$ ), however, feature the steepest slope in the dissociation phase (most clearly observed after $180 \mathrm{~s}$ ), suggesting that $k_{\mathrm{d}}$ has changed, hence supporting our hypothesis.

\section{Discussion}

In terms of GT activity inhibition, the studied compounds can be ranked as follows from the best active to the least active $62>44>43>21>61=57>56$ (Fig. 6) [5]. Compounds 21 and 57 differ in the dipeptide moiety LAla-DGlu in 57. Compound 21 showed better enzymatic inhibition and higher MtgA binding enhancement to moenomycin A (cooperative effect) than compound 57 . This shows that the peptide affects at least partially the binding to the acceptor site. Compounds 62 and 57 both have a dipeptide moiety but differ in the phosphate linker, 57 has a monophosphate group and 62 has a phosphoglycerate, a mimic of the pyrophosphate of the substrate. 62 causes higher increase in GT binding to moenomycin A than 57 . This difference suggests that the phosphoglycerate group may be an important determinant for binding to the acceptor site. Compound 43 is a monosaccharidephosphoglycerate with GT inhibition activity comparable to 44 and 62 but did not show any enhancing effect on moenomycin A binding, yet only inhibits binding at higher concentration. These results suggest that 43 may have a poor affinity for the acceptor site and higher binding affinity to the donor site. It is noticeable that the four lipid II analogs with disaccharide structure (inhibitors 21, 44, 57 and 62) were the ones enhancing MtgA binding at low concentrations. Therefore it seems likely that the disaccharide moiety is more important for the affinity towards the acceptor site than for the affinity towards the donor site. A disaccharide compound such as 44 , without a peptide and with phosphoglycerate-lipid group, seems to be the best ligand to target both the donor and acceptor sites. Co-crystallization of a GT with this compound would provide valuable information to better understand the catalytic mechanism and could help in the design of new inhibitors.

Most of the crystal structures of GT in apo form or in complex with moenomycin $A$, show an unstructured or mobile region in the jaw domain between donor and acceptor sites. This region (Ser121-Gly130) located between conserved motifs 1 and 2 was proposed to have an important role in the binding of the acceptor substrate [16]. We propose that the disaccharide analogs (or lipid II) bind preferentially to the acceptor site and stabilize the mobile region which induces local structure rearrangement in the donor site increasing its affinity for moenomycin A (or the second lipid II). This mechanism may be particularly critical during the initiation stage (lipid II in the donor site). The allosteric activation of binding in the donor site could also apply to the elongating glycan chain and would play an important role in the processive elongation mechanism. Consequently, after each catalytic cycle the translocation of the product from the acceptor site to the donor site is concomitant with the unfolding or movement of the mobile region, 



Fig. 6. Lipid II and glycosyltransferase inhibitors mimicking lipid II [5].

which contribute to this process before a new lipid II molecule binds and stabilizes it again to start a new catalytic reaction.

The bi-substrate reaction of the GT at initiation stage of polymerization makes distinction between the affinities for the substrate of the donor site and acceptor site complex. Moenomycin A binds to the donor site of the GT and is proposed to mimic the growing chain. Lipid II binds to both sites as would do its analogs. The concentration dependent effect of lipid II analogs on moenomycin A binding indicates the presence of allosteric activation of the donor site following ligand binding to the acceptor site. Due to structural similarities between lipid II and the investigated inhibitors and based on the obtained data we expect the same behavior for lipid II itself. Solubility problems however, resulting in a high spread of the binding responses, prevented a thorough investigation for this compound.

This effect has great impact on the catalytic mechanism and could explain the role of the mobile region between the two sites.
The binding of lipid II to the acceptor site could induce local structural rearrangement, which also affects the donor site via the mobile region. During elongation phase, stabilization of the mobile region would increase binding to the elongating chain and contribute to the processive polymerization. After each catalytic cycle, product translocation from acceptor to donor site is assisted by higher affinity of the pyrophosphate-lipid moiety (larger positively charged patches inside the donor site) and the mobility of the unstructured region, concomitant with the vacancy of the acceptor site.

So far we showed enhancement of MtgA binding to moenomycin $A$, which is supposed to mimic the growing glycan chain. Further studies using other approaches are needed to better characterize the postulated cooperativity mechanism by investigating the interaction between lipid II and MtgA and eventually other GTs. To be biologically relevant for peptidoglycan synthesis, such an enhancement should also increase the affinity of the donor 



$\begin{array}{lll}\diamond \text { Inhibitor } 21 & \diamond \text { Inhibitor } 62 \diamond \text { Inhibitor } 43 \diamond \text { Inhibitor } 61 \text { O No inhibitor } \\ \diamond \text { Inhibitor } 44 & \diamond \text { Inhibitor } 57 \diamond \text { Inhibitor } 56 \Delta \text { Lipid II }\end{array}$

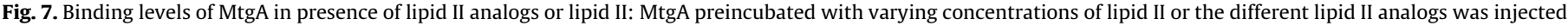

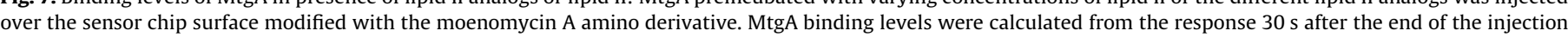


(d) lipid II.

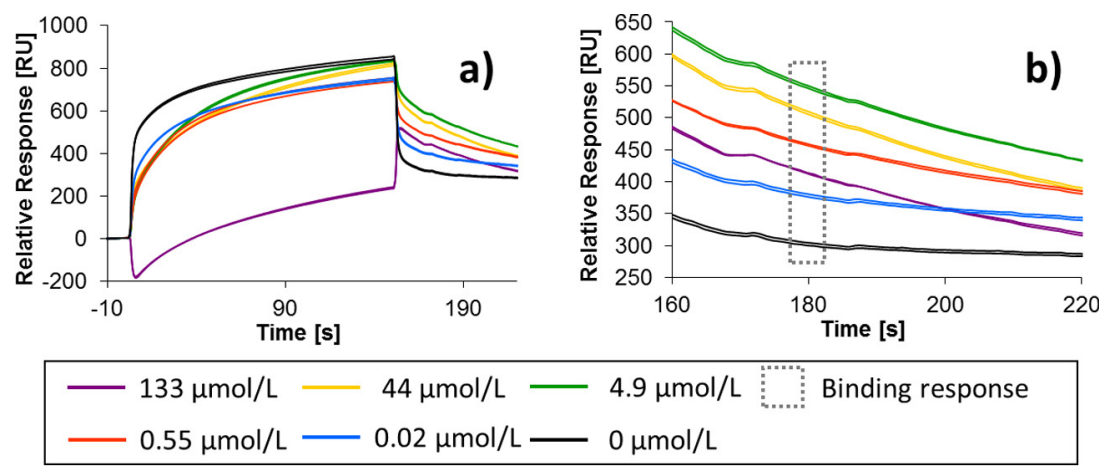

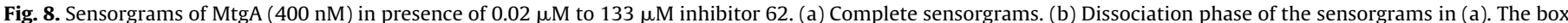

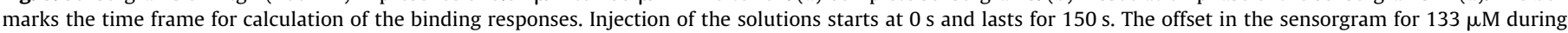
injection is due to a slight mismatch in DMSO content between the injected solution and the running buffer.

site of MtgA to the growing glycan chain itself. SPR experiments with the tetrasaccharide lipid IV tethered to the sensor chip surface instead of moenomycin A could prove beneficial here.

\section{Acknowledgments}

This work was supported by the Fond de la Recherche Fondamentale Collective FRFC no. 2.4543.12, the Belgian Science Policy programs IAP-VII iPROS project (no. P7/44), FRIA fellowship to ID and the Belgian Science Policy return grant to AD. Mohammed Terrak is research associate of the F.R.S-FNRS. We thank Professor Heiko Hayen for valuable advice during development of the isolation procedure for moenomycin A and Eric Sauvage for help in preparation of Fig. 1.

\section{References}

[1] Sauvage E, Kerff F, Terrak M, Ayala JA, Charlier P. The penicillin-binding proteins: structure and role in peptidoglycan biosynthesis. FEMS Microbiol Rev 2008;32:234-58.
[2] Huang SH, Wu WS, Huang LY, Huang WF, Fu WC, Chen PT, et al. New continuous fluorometric assay for bacterial transglycosylase using Forster resonance energy transfer. J Am Chem Soc 2013;135:17078-89.

[3] Gampe CM, Tsukamoto H, Doud EH, Walker S, Kahne D. Tuning the moenomycin pharmacophore to enable discovery of bacterial cell wall synthesis inhibitors. J Am Chem Soc 2013;135:3776-9.

[4] Shih HW, Chen KT, Chen SK, Huang CY, Cheng TJ, Ma C, et al. Combinatoria approach toward synthesis of small molecule libraries as bacterial transglycosylase inhibitors. Org Biomol Chem 2010;8:2586-93.

[5] Dumbre S, Derouaux A, Lescrinier E, Piette A, Joris B, Terrak M, et al. Synthesis of modified peptidoglycan precursor analogues for the inhibition of glycosyltransferase. J Am Chem Soc 2012;134:9343-51.

[6] Gampe CM, Tsukamoto H, Wang TS, Walker S, Kahne D. Modular synthesis of diphospholipid oligosaccharide fragments of the bacterial cell wall and their use to study the mechanism of moenomycin and other antibiotics. Tetrahedron 2011;67:9771-8.

[7] Welzel P. Syntheses around the transglycosylation step in peptidoglycan biosynthesis. Chem Rev 2005:105:4610-60.

[8] Ostash B, Walker S. Moenomycin family antibiotics: chemical synthesis, biosynthesis, and biological activity. Nat Prod Rep 2010;27:1594-617.

[9] Terrak M, Sauvage E, Derouaux A, Dehareng D, Bouhss A, Breukink E, et al. Importance of the conserved residues in the peptidoglycan glycosyltransferase module of the class A penicillin-binding protein 1b of Escherichia coli. J Biol Chem 2008;283:28464-70.

[10] Schwartz B, Markwalder JA, Seitz SP, Wang Y, Stein RL. A kinetic characterization of the glycosyltransferase activity of Eschericia coli PBP1b and development of a continuous fluorescence assay. Biochemistry 2002;41:12552-61. 
G Model

BCP-12134; No. of Pages 10

10

D. Bury et al./ Biochemical Pharmacology $x x x$ (2014) $x x x-x x x$

[11] Perak M, Gosh TK, van Heijenoort J, Van Beeumen J, Lampilas M, Aszodi J, et al. The catalytic, glycosyl transferase and acyl transferase modules of the cell wall peptidoglycan-polymerizing penicillin-binding protein lb of Escherichia coli. Mol Microbiol 1999;34:350-64.

[12] Fraipont C, Sapunaric F, Zervosen A, Auger G, Devreese B, Lioux T, et al. Glycosyl transferase activity of the Escherichia coli penicillin-binding protein lb: specificity profile for the substrate. Biochemistry 2006;45: 4007-13.

[13] Perlstein DL, Zhang Y, Wang TS, Kahne DE, Walker S. The direction of glycan chain elongation by peptidoglycan glycosyltransferases. J Am Chem Soc 2007;129:12674-75.

[14] Perlstein DL, Wang TS, Dour EH, Kane D, Walker S. The role of the substrate lipid in processive glycan polymerization by the peptidoglycan glycosyltransferases. J Am Chem Soc 2010;132:48-9.

[15] hovering AL, de Castro LH, Rim D, Strynadka NC. Structural insight into the transglycosylation step of bacterial cell-wall biosynthesis. Science 2007;315: 1402-5.

[16] hovering AL, De Castro L, Strynadka NC. Identification of dynamic structural motifs involved in peptidoglycan glycosyltransfer. J Mol Biol 2008;383:16777.

[17] Sung MT, tai YT, Huang CY, Chou LY, Shin HW, Cheng WC, et al. Crystal structure of the membrane-bound bifunctional transglycosylase PBP1b from Escherichia coli. Proc Natl Aced Sci USA. 2009;106:8824-9.

[18] Heaslet H, Shaw B, Mistry A, Miller AA. Characterization of the active site of $S$. aureus monofunctional glycosyltransferase (Mtg) by site-directed mutation and structural analysis of the protein complexes with moenomycin. J Struct Biol 2009;167:129-35.

[19] Huang CY, Shin HW, Lin LY, Then YW, Chen TJ, Cheng WC, et al. Crystal structure of Staphylococcus aureus transglycosylase in complex with a lipid II analog and elucidation of peptidoglycan synthesis mechanism. Proc Natl Aced Sci USA. 2012;109:6496-501.

[20] Yuan Y, Barrett D, Chang Y, Kane D, Sliz P, Walker S. Crystal structure of a peptidoglycan glycosyltransferase suggests a model for processive glycan chain synthesis. Proc Natl Aced Sci USA. 2007;104:5348-53.
[21] Stembera K, Vogel S, Buchynskyy A, Ayala JA, Welzel P. A surface plasmon resonance analysis of the interaction between the antibiotic moenomycin $A$ and penicillin-binding protein Ib. ChemBioChem 2002;3:559-65.

[22] Nicholas RA, Lawson DR, Schultz DE. Penicillin-binding protein 1B from Escherichia coli contains a membrane association site in addition to its transmembrane anchor. J Biol Chem 1993;268:5632-41.

[23] Breukink E, van Heusden HE, Vollmerhaus PJ, Swiezewska E, Brunner L, Walker $\mathrm{S}$, et al. Lipid II is an intrinsic component of the pore induced by nisi in bacterial membranes. J Biol Chem 2003;278:19898-903.

[24] Bury D, Müller-Seitz E, Hayen H, Putz M. Isolierung vo Moenomycin A aus dem Mastförderer Flavomycin zur Synthese vo Derivaten für einen SPRAssay. Weitere erweiterte Posterbeiträge vow Lebensmittelchemikertag 2012.Lebensmittelchemie 2013;67:2-7. http://dx.doi.org/10.1002/lemi. 201390005.

[25] Stembera K, Buchynskyy A, Vogel S, Knoll D, Osman AA, Ayala JA, et al. Moenomycin-mediated affinity purification of penicillin-binding protein $1 \mathrm{~b}$. ChemBioChem 2002;3:332-40.

[26] Esterbauer H. Über die Autoxydation vo Linolsäure-methylester in Weser III: Chromatographische Auftrennung der wasserlöslichen Reaktionsprodukte. Pete Seifen Anstrichm 1968;70:1-4.

[27] Perak M, Nguyen-Distèche M. Kinetic characterization of the monofunctional glycosyltransferase from Staphylococcus aureus. J Bacteriol 2006;188:252832.

[28] Hennig L, Findeisen M, Welzel P, Haessner R. ${ }^{1} \mathrm{H}$ NMR spectroscopic studies of the moenomycins. Man Reason Chem 1998;36:615-20.

[29] Morton TA, Myszka DG, Chaiken IM. Interpreting complex binding kinetics from optical biosensors: a comparison of analysis by linearization, the integrated rate equation, and numerical integration. Anal Biochem 1995;227: $176-85$.

[30] Rich RL, Myszka DG. Survey of the year 2007 commercial optical biosensor literature. J Mol Recognit: JMR 2008;21:355-400.

[31] Wallhausser KH, Nesemann G, Prove P, Steigler A. Moenomycin, a new antibiotic I. Fermentation and isolation. Antimicrob Agents Chemother 1965;5:734-6.

Please cite this article in press as: Bury D, et al. Positive cooperativity between acceptor and donor sites of the peptidoglycan glycosyltransferase. Biochem Pharmacol (2014), http://dx.doi.org/10.1016/j.bcp.2014.11.003 